\title{
Dependence of Order and Dynamics in Polymers and Elastomers under Deformation Revealed by NMR Techniques
}

\author{
S. STAPF* AND S. KARIYO \\ Lehrstuhl für Makromolekulare Chemie \\ and Magnetic Resonance Center MARC, ITMC, RWTH Aachen, Germany

\begin{abstract}
Uniaxial stretching and swelling are considered as two limiting cases of deformations of elastomers. Under both conditions, the molecular dynamics is changed with respect to the behavior that describes the undisturbed, equilibrium elastomer. Particularly the spectrum of segmental motions, which reveals itself in the frequency-dependence of the longitudinal NMR relaxation time, is discussed in this study, but also order effects expressed via the dipolar coupling strength are investigated. For stretched elastomers, a significant change of the relaxation dispersion is found for three different types of rubber; it is a consequence of a change of the mode spectrum of segmental motions that becomes obvious at low frequencies (below $1 \mathrm{MHz}$ at room temperature). In swollen elastomers, on the other hand, a cross-over towards a behavior expected for semi-dilute solutions is found, and a comparison to solutions of uncross-linked polymers reveals a significant effect of the cross-links only in the $\mathrm{kHz}$ range. A much more pronounced difference between elastomers and polymer solutions, however, is found from double-quantum encoded NMR measurements where the residual order introduced by the presence of permanent cross-links is maintained even in the presence of solvent.
\end{abstract} \\ PACS numbers: 81.05.Lg, 82.35.Lr, 76.60.-k, 61.25.Hq, 36.20.Ey, \\ 81.40.Lm
}

\section{Introduction}

A major issue in polymer science is to gain an understanding of the molecular dynamics which is of interest both from the theoretical point of view and

*corresponding author; e-mail: sstapf@mc.rwth-aachen.de 
with the goal of establishing a relationship with macroscopic material properties. Just as macroscopic quantities are time- and frequency-dependent, the molecular dynamics needs to be described over many orders of magnitude in frequency so that a complete picture of the motions of chain molecules can be achieved.

Polymer molecules, with molecular weights $M_{\mathrm{w}}$ above a characteristic threshold $M_{\mathrm{c}}$, experience multiple entanglements which slow down their mobility but leave them with basic properties of a liquid in the melt state; in particular, the mean-squared displacement $\left\langle R^{2}\right\rangle$ for a segment, or the center-of-mass, diverges for long times. Introducing permanent cross-links between the chains, such as sulfur-bridges after vulcanization in natural rubber, obviously changes the mechanical behavior on long time scales drastically, as the macroscopic sample now is a static object and $\left\langle R^{2}\right\rangle$, for any given segment, or nucleus, in the chain is finite even for long times. At high frequencies, however, the qualitative difference between a melt and a rubber disappears. This can be best understood as the regime when the segmental motion amplitudes are so small that they are not affected by the presence of cross-links. It thus makes sense to compare elastomers with the same formalism as melts, but keeping in mind that deviations will occur above a threshold timescale.

Introducing cross-links is only one possibility to restrict the mobility of polymer chains relative to the equilibrium state of the melt. Deforming the network out of its original shape certainly affects the local environment of the molecules, and the purpose of this study is to determine whether the dynamical features are influenced as well. Rather than measuring the mean-squared displacements $\left\langle R^{2}(t)\right\rangle$ directly, which is possible by NMR methods but limited to time scales even longer than those of interest, the frequency dependence of the longitudinal relaxation time, $T_{1}(\omega)$, is often used to describe polymer dynamics. It is coupled to the time-dependence of the autocorrelation function of spin interactions, $G(t)$, via the spectral density function of reorientational motions, $\mathcal{I}(\omega)$ :

$$
T_{1}^{-1}(\omega)=C[\mathcal{I}(\omega)+4 \mathcal{I}(2 \omega)],
$$

where

$$
\mathcal{I}(\omega)=\int_{-\infty}^{\infty} G(t) \mathrm{e}^{-\mathrm{i} \omega t}
$$

and $C$ is a geometry- and interaction-dependent constant prefactor.

Because of the simultaneous occurrence of translation and rotational motion in chain segments, $\left\langle R^{2}(t)\right\rangle$ and $T_{1}(\nu)$ are two alternative measures of molecular motion, albeit not providing identical information.

For the case of polymer melts and concentrated solutions, the movements of a given entangled chain are restricted by topological constraints of their surrounding chains. The mathematical formulation of the local drag needs to be supplemented by a model taking these topological restrictions into account. This is the role of the tube model introduced and developed by Doi and Edwards [1] together 
with the reptation motion suggested by de Gennes [2]. The tube/reptation model, however, considers the dynamics of the polymer chain in the presence of fixed obstacles. Therefore, Schweizer [3] proposed the renormalized Rouse formalism in which the entanglement effects were taken into account by a generalized Langevin equation applying a so-called memory function term. Based on the renormalized Rouse formalism, Kimmich and Fatkullin [4] predicted a power law dependence of the NMR spin-lattice relaxation time as a function of Larmor frequency for describing the dynamics of polymer melts. Three different regions of power laws describing the relaxation dispersion for the chain modes dynamics of polymers can be distinguished [5]. They have been identified and confirmed experimentally for a number of polymers, among them polybutadiene and polydimethylsiloxane $[6,7]$. The exponents for polyisoprene were found to be somewhat different [8-10], but a power-law was still observed. The same is true for elastomers and networks consisting, for instance, of chains of polyisoprene or polybutadiene [8, 9, 11-13]. In semi-dilute solutions, or polymers with molecular weights below the critical value, chains are not affected by entanglements and the frequency dependence changes toward Rouse behavior, i.e. a logarithmic relationship [14]:

$$
T_{1}^{-1}(\omega) \propto \ln \left(\frac{1}{\omega \tau_{\mathrm{s}}}\right)
$$

where $\tau_{\mathrm{s}}$ is an average (local) segment reorientation time. Experimental evidence about $\tau_{\mathrm{s}}$ is obtained from the temperature minimum of the relaxation time. Assuming that short-time reorientations can indeed be described by an average time, corresponding to an exponential correlation function, the spectral density will be a Lorentzian:

$$
\mathcal{I}(\omega)=\frac{\tau_{\mathrm{s}}}{1+\omega^{2} \tau_{s}^{2}},
$$

and the minimum of $T_{1}$ is found when the condition $\omega \tau_{\mathrm{c}}=0.62$ is fulfilled.

The prerequisite for discussing molecular motion in terms of $T_{1}(\omega)$ is a technique that gives access to the frequency-dependence of relaxation times. The NMR field-cycling method is a technical realization that accesses Larmor frequencies over $3-4$ orders of magnitude [15-17].

At the low end of the frequency spectrum, the transverse relaxation time $T_{2}$ supplies a different type of information but without giving direct access to the spectrum of molecular motion. Its value is dominated by the zero-frequency contribution of the spectral density function of motions, and as such it is affected by the total effect of reorientations at all frequencies to a limiting value which is given by the lifetime of the magnetization coherence itself. Another approach to determine dynamics is the investigation of order phenomena which affect the coupling between different nuclear spins: much as relaxation measures the process at which these correlations decay, multi-pulse NMR techniques are applied as means to determine molecular order which remains after partial pre-averaging up 
to a certain time scale, or incorporate variable delays during which molecular order is allowed to evolve. The encoding and reconversion of double-quantum coherence is one of those methods that has been successfully applied to soft matter and semi-solid materials $[18,19]$.

In this study, the influence of swelling on the frequency dependence of the longitudinal relaxation time $T_{1}$ is investigated for the first time, and is compared to equivalent solutions of uncross-linked polymers, supplemented by measurements of $T_{2}$ and coupling constants obtained from double quantum experiments for the same systems.

\section{Experimental}

Natural rubber (NR) and butadiene rubber (BR) samples were provided by Dunlop GmbH, Germany. They were vulcanized into sheets of $2 \mathrm{~mm}$ thickness at $160^{\circ} \mathrm{C}$ with $3 \mathrm{phr}$ (per hundred rubber) $\mathrm{ZnO}, 2 \mathrm{phr}$ stearic acid, and equal amounts of sulfur and N-tert-butyl-2-benzothiazole sulfenamide (TBBS) as accelerator of between 1 and $4 \mathrm{phr}$ for BR (denotes as BR $1 / 1 \ldots \mathrm{BR} 4 / 4$ ) or between 1 and $7 \mathrm{phr}$ for NR (denoted as NR $1 / 1 \ldots$ NR $7 / 7$ ), respectively. Experiments on NR were carried out on unfilled samples. BR rubber BR $1 / 1$ for the deformation experiments was modified by the addition of $30 \mathrm{phr}$ of carbon black type N660. As polydimethylsiloxane (PDMS) rubber sample, biomedical grade silicone sheeting from Specialty Manufacturing Inc., Saginaw, MI, USA was used.

The polyisoprene melts used for comparison were purchased from Polymer Standards Service, Mainz, Germany, and were characterized as 95\% 1,4- and 5\% 3,4-linked. Samples used for this study possessed number-averaged molecular weights of $\overline{M_{n}}=287000$ and $\overline{M_{n}}=709000$, respectively, and the polydispersity $M_{w} / M_{n}$ was better than 1.10. All samples were stored at $277 \mathrm{~K}$ between experiments. Toluene-d8 (purity 99.6\%) and ethanol-d6 (purity 98\%) for the swelling experiments were obtained from Sigma-Aldrich Fine Chemicals and used without further purification; measurements of the pure solvents were performed at the relaxometer and revealed no detectable proton signal.

For the uniaxial deformation study, NR1/1 and BR1/1 samples were used as they allowed the highest values of stretching ratio, $\lambda$. A single band of each rubber sample of approximately $3-4 \mathrm{~mm}$ width and $2 \mathrm{~mm}$ thickness was prepared and was either stretched in a U-shape about a hard plastic plate or wound tightly around an epoxy resin rod. The orientations of stretching direction relative to the direction of the external magnetic field, $B_{0}$, were parallel (U-shape) and almost perpendicular (at angles between 70 and 80 ) to $B_{0}$ (winding around the epoxy rod). Both spacer materials did not contribute to the acquired NMR signal because of their short signal duration. The maximum stretching ratios which could be achieved in this study were $\lambda \approx 2$ for silicone rubber, $\lambda \approx 3.5$ for butadiene rubber BR $1 / 1$, and $\lambda \approx 7$ for natural rubber NR $1 / 1$. 
For the swelling experiments, NR samples were investigated under equilibrium swelling conditions, i.e. by immersing pieces of rubber of about 100-150 mg weight each into a mixture of toluene-d8 (good solvent) and ethanol-d6 (bad solvent) and performing the experiments in the liquid-filled sealed tube at least $48 \mathrm{~h}$ after beginning of the sample preparation. Ethanol as a bad solvent for rubber led to a weight increase of only about $4 \%$ upon immersion of the sample. Higher degrees of swelling were obtained by preparing appropriate mixtures with toluene-d8 (see Fig. 1). The same weight of liquid was subsequently added to the polyisoprene melt samples to achieve a comparable dilution; in some cases, the solvent mixture was replaced by pure toluene-d8.

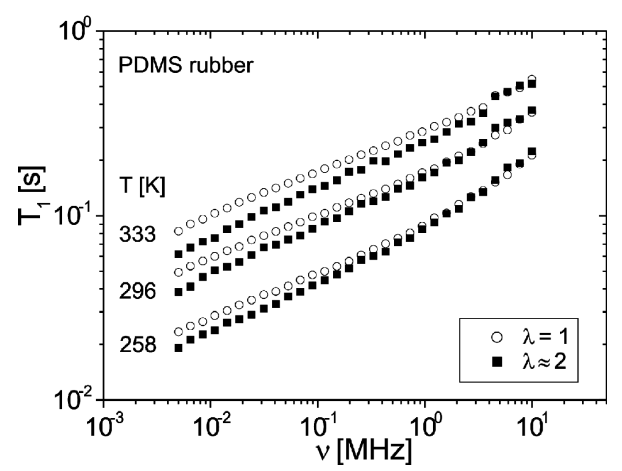

Fig. 1. Dispersion (dependence on Larmor frequency) of the ${ }^{1} H$ longitudinal relaxation time $T_{1}$ for polydimethylsiloxane elastomer unstretched $(\lambda=1)$ and at a uniaxial deformation at $\lambda \approx 2$ parallel to the magnetic field. Measurements were taken out at the temperatures indicated.

Relaxometry data were obtained on a commercial Stelar fast field cycling relaxometer (Stelar s.r.l., Mede, Italy) on which proton longitudinal relaxation times were determined at Larmor frequencies between $5 \mathrm{kHz}$ and $10 \mathrm{MHz}$. Experiments on uniaxially deformed samples were carried out at temperatures within a maximum range of $223 \mathrm{~K}$ to $348 \mathrm{~K}$, while swollen samples and solutions were measured at $296 \mathrm{~K}$. Decays were found to be monoexponential over more than one decade in all cases. Additional room-temperature $(296 \mathrm{~K})$ proton $T_{1}$ measurements as well as $T_{2}$ experiments employing Carr-Purcell-Meiboom-Gill (CPMG) pulse sequences, and double quantum built-up curves were recorded at a $4.7 \mathrm{~T}$ magnet equipped with a Bruker DSX-200 spectrometer.

\section{Results and discussion}

\subsection{Uniaxial deformation of elastomers}

The intermediate regime of molecular motion, designated "region II" [20] and corresponding to the low-mode number limit of the segment fluctuations, is 
commonly observed in polymer melts within the experimentally accessible range of low-field NMR relaxometry, i.e. between $\mathrm{kHz}$ and $\mathrm{MHz}$. For linear PDMS, the relationship $T_{1}(\nu) \propto \nu^{\gamma}$ with $\gamma \approx 0.24$ was found, and was confirmed for cross-linked PDMS [7]. Figure 1 presents the effect of uniaxial deformation at $\lambda \approx 2$ parallel to $B_{0}$ on PDMS rubber at three different temperatures. For $T=333 \mathrm{~K}$, an increase of the slope of the dispersion relation from $\gamma=0.24$ to $\gamma=0.28$ is clearly found; $T_{1}$ values above a divergence frequency of several $\mathrm{MHz}$ cannot be distinguished. For lower temperatures, a similar effect occurs but it is less obvious due to the shift of the divergence point towards lower Larmor frequencies, thus reducing the relative difference between the two datasets at the lowest detected Larmor frequency of $5 \mathrm{kHz}$ which was limited in all experiments by the presence of the Earth's magnetic field and uncompensated laboratory background fields.

PDMS rubber represents a suitable test case that demonstrates the influence of deformation on the relaxation behavior. The very same sample types have been investigated with respect to order parameters at deformation [21]. For the comparatively low elongation ratio of $\lambda=2$, strain-induced crystallization is not expected so that the change of the dispersion slope indeed reflects a change in the mode distribution of the segment dynamics.

A second test case is natural rubber that has been extensively studied with a range of NMR techniques by many research groups. Considerably higher degrees of deformation can be achieved easily with unfilled, weakly cross-linked NR, but it is known that above $\lambda \approx 3$, crystallization sets in this polymer [22, 23]. The effect of deformation on the transverse relaxation time, $T_{2}$, has been investigated [24], and angular dependent order parameters were obtained from double quantum encoding techniques [25]. In Fig. 2, the effect of stretching at $\lambda \approx 7$ on a sample of NR $1 / 1$

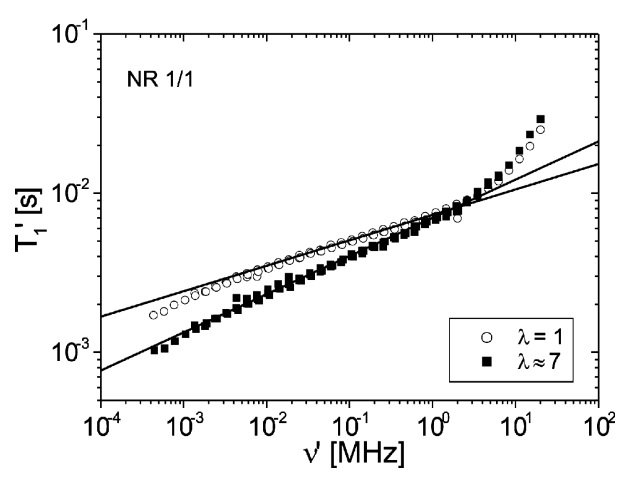

Fig. 2. Dispersion (dependence on Larmor frequency) of the ${ }^{1} H$ longitudinal relaxation time $T_{1}$ for natural rubber NR $1 / 1$ unstretched $(\lambda=1)$ and at a uniaxial deformation at $\lambda \approx 7$ perpendicular to the magnetic field. Measurements were carried out at temperatures between $275 \mathrm{~K}$ and $333 \mathrm{~K}$ and are plotted on a master curve relative to a reference temperature of $296 \mathrm{~K}$. Solid lines indicate best fits to the frequency dependences $T_{1} \propto \nu^{0.16}(\lambda=1)$ and $T_{1} \propto \nu^{0.24}(\lambda \approx 7)$, respectively. 
is plotted by shifting the relaxation curves in horizontal and vertical direction by an equivalent shifting factor $a_{T}$ that is derived from the Wiliams-Landel-Ferry (WLF) equation. The shift factor follows the relation $\log a_{T}=-C_{1}\left(T-T_{0}\right) /\left(C_{2}+\right.$ $\left.T-T_{0}\right)$, where the constants $C_{1}, C_{2}$ were determined empirically with $T_{0}$ set to $296 \mathrm{~K}[10]$. The curves were reconstructed from original data obtained between $275 \mathrm{~K}$ and $333 \mathrm{~K}[9,11]$. While $\gamma=0.16$ for the undeformed rubber - a value that is somewhat lower than the one found for other polymers but is still assigned to region II - an increase to $\lambda=0.24$ is observed for stretching at $\lambda \approx 7$, and intermediate slopes for smaller stretching ratios. The magnitude of this effect is certainly significant. Because of the small available sample volume, an angular dependence of the $T_{1}$ dispersion could not be investigated, but deformation parallel and perpendicular to the magnetic field axis revealed indistinguishable results. Likewise, a direct influence of the presence of nanocrystallites to the relaxation behavior could be ruled out because of the dead-time chosen for the receiver, but even indirect influences due to mixing by spin diffusion is unlikely [11], while the reduction in mobility of chains in the amorphous phase by the presence of a crystalline phase might indeed play a role, although the similarity to the effect found for PDMS rubber that does not crystallize speaks against this. Again, the relaxation effect can directly be translated into a change of the shape of the segmental motion's autocorrelation function, as only intramolecular contributions were identified as the responsible mechanism for the observed ${ }^{1} H$ relaxation [10].

The cross-over to "region III" at the low-frequency end of the data, just being indicated in the NR measurements that could not be extended towards much higher temperatures due to a possible decomposition of the network, is of particular interest in the context of deformation studies. Here, segment oscillations on a larger length scale become relevant that require coherent motions of larger chain units and are restricted by geometrical restrictions by the surrounding chains (often called the "tube"), and intermolecular contributions were suggested to gain importance in this regime [20]. In order to test the hypothesis whether the cross-over between regions II and III is shifted due to deformation, butadiene rubber was chosen as a suitable material because it brings the dynamics in region III into reach within the experimentally accessible range of frequencies and temperatures as has been shown for the pure melt [26]. Figure 3 combines results obtained between $223 \mathrm{~K}$ and $348 \mathrm{~K}$ shifted relative to a reference temperature of $296 \mathrm{~K}$; the shift factors could be described well by the WLF formalism with the exception of the lowest temperatures, which do not have to be considered here because they refer to the high-frequency branch in Fig. 3.

Just as for PDMS rubber and NR, a slight increase in $\gamma$ in region II is found for BR. A similar effect can be fitted for the slope in region III which is $\gamma=0.50$ for the unstretched sample in accordance to theoretical expectations [20], but the difference is less obvious due to the narrower range of region III which is bounded by the experimental low-field limit. What is remarkable, however, is the shift of 


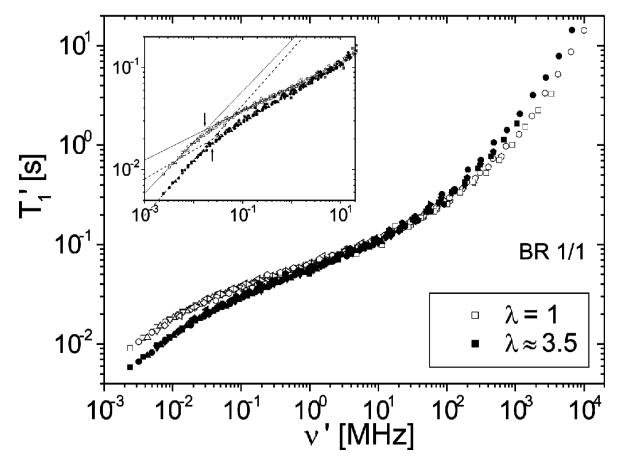

Fig. 3. Dispersion (dependence on Larmor frequency) of the ${ }^{1} H$ longitudinal relaxation time $T_{1}$ for butadiene rubber BR $1 / 1$ unstretched $(\lambda=1)$ and at a uniaxial deformation at $\lambda \approx 3.5$ perpendicular to the magnetic field. Measurements were carried out at temperatures between $223 \mathrm{~K}$ and $348 \mathrm{~K}$ and are plotted on a master curve relative to a reference temperature of $296 \mathrm{~K}$. The inset magnifies the low-frequency part of the data, arrows indicate cross-overs between regions II and III of the dispersion relation (see the text).

the cross-over point between regions II and III (indicated by arrows in the inset that magnifies the low-field relaxation) towards higher frequencies for the stretched BR sample. One can interpret this finding by the restrictions due to neighboring chains being experienced at low frequencies, corresponding to large motion amplitudes. When the whole sample is stretched, the local environment of each chain is also changed. The amplitude of motion of a chain required to interact with its neighbors becomes smaller. Therefore, the transition point between regions II and III shifts to shorter motional amplitudes, i.e. shorter times and, therefore, higher frequencies, which is confirmed by the experimental data.

In conclusion, uniaxial deformation of elastomers has been found to affect the autocorrelation function of segmental reorientations in such a way that a more pronounced low-field frequency dependence is observed for all materials under study, while high-field relaxation remains essentially unaffected.

\subsection{Swelling of elastomers in solvents}

Although being of a completely different nature, swelling of elastomers can be regarded as another limiting case of sample deformation. The dominating contribution to the relaxation behavior, however, is not the change in polymer chain distances but rather the increased chain mobility due to the addition of solvents that is found for cross-linked elastomers the same way as for polymer solutions. The increase in mobility is directly revealed in a change of the segmental reorientation time, $\tau_{\mathrm{s}}$ because of the lubricating effect of the solvent. This has the same effect as a change of temperature which has been described above in the Introduction: at a particular temperature, a minimum of $T_{1}$ is found where the 
condition $\omega \tau_{\mathrm{s}} \approx 0.62$ is fulfilled, assuming that $\tau_{\mathrm{s}}$ is a well-defined quantity and a wide range of relevant correlation times does not exist in the range of interest. Figure 4 demonstrates the occurrence of the temperature minimum for cross-linked $\mathrm{NR}$ in the swollen state as a function of solvent concentration. At the minimum, the condition requires that $\tau_{\mathrm{s}} \approx 5 \times 10^{-10} \mathrm{~s}$ because measurements were carried out at $\nu=200 \mathrm{MHz}$. Despite the scatter in the data, a general trend is visible that for higher cross-link densities, the corves are shifted towards the right, i.e. a larger amount of solvent is required to make $\tau_{\mathrm{s}}$ assume the mentioned value. This is in agreement with the finding that $\tau_{\mathrm{s}}$ increases with the degree of cross-linking and that its change corresponds to the shift of the glass transition temperature in elastomers [8]. For linear polyisoprene in solution, results qualitatively similar to those shown in Fig. 4 were obtained.

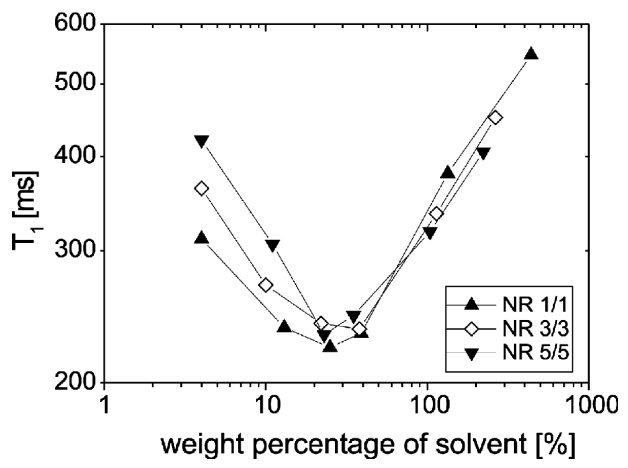

Fig. 4. Dependence of the ${ }^{1} H$ longitudinal relaxation time $T_{1}$ at a Larmor frequency of $200 \mathrm{MHz}$ for natural rubber of different cross-linking density at equilibrium swelling in ethanol/toluene mixtures.

The change of relaxation times on addition of solvent becomes much more pronounced when experiments are performed at low frequencies. The expected behavior for the relaxation dispersion in solutions has been demonstrated for PDMS in $[7,20]$ : a cross-over from a series of power-law relations $T_{1} \propto \nu^{\gamma}$ to a logarithmic dependence $T_{1}^{-1}(\omega) \propto \ln \left(1 / \omega \tau_{\mathrm{s}}\right)$ is seen with increasing dilution. In Fig. 5, dispersion curves for NR $1 / 1$ at equilibrium swelling are compared with solutions of polyisoprene of $\overline{M_{n}}=709000$ in exactly the same amount of solvent. This comparison, however, must remain qualitative because the cross-over from power-law to logarithmic (Rouse) behavior, equivalent to the point where the entanglement length becomes equal to the chain length, necessarily depends on the molecular weight. Common to the measurements is a dramatic increase in $T_{1}$ which assumes a factor of 100 or more at the lowest observed frequency of $10 \mathrm{kHz}$. The high-frequency values of Fig. 4 are included into Fig. 5; their variation is small by comparison. As was suggested by Doi and Edwards [1], a transition from the melt or the concentrated solution toward a semi-dilute solution has the consequence 
that physical entanglements do not affect the molecular mobility any longer. In the case of the elastomers, chemical cross-links remain and restrict the mobility of chains which should become most prominent at high swelling ratios and low frequencies. Indeed, while at a weight increase of $439 \%$ the dispersion curve for the polyisoprene solution is already flat, a weak residual frequency dependence is still found for the elastomer. Still, the relaxation dispersion in the observed range of $10^{4} \mathrm{~Hz}$ to $10^{8} \mathrm{~Hz}$ must be called similar in comparing cross-linked and uncross-linked polymer chains. What is perhaps more striking is the sensitivity of the longitudinal relaxation at low fields to small amounts of solvent. Even at $4 \%$ weight increase the change of $T_{1}$ at low frequencies is significant, and at $13 \%$ it amounts to an increase by a factor of about 3 . The technique is thus very sensitive to the detection of solvent effects, but almost unable to identify the presence of cross-links.
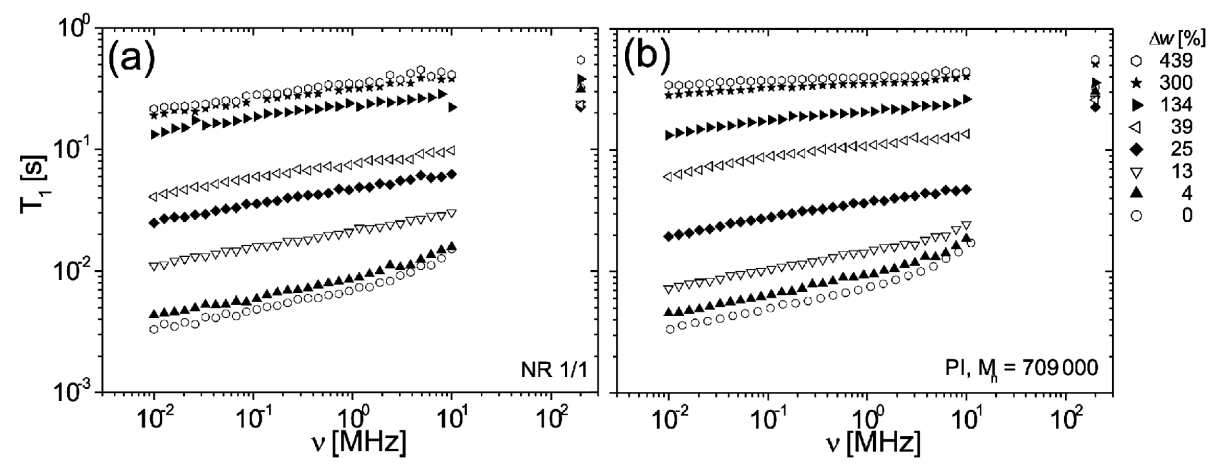

Fig. 5. (a) Dispersion (dependence on Larmor frequency) of the ${ }^{1} H$ longitudinal relaxation time $T_{1}$ for natural rubber NR $1 / 1$ at equilibrium swelling in ethanol/toluene mixtures. Numbers to the right of the plot indicate weight percentage of solvent relative to the dry polymer weight. (b) Data obtained for solutions of polyisoprene of a number-averaged molecular weight of 709000 at the same relative amounts of solvent as in $(\mathrm{a})$.

While $T_{1}$ was always found to be monoexponential, the transverse relaxation time $T_{2}$ can indeed be decomposed into two or more contributions which have a known physical significance. In principle, biexponential decays can be assigned to the chain ends and the central parts of the molecular chains in melts [27]. In cross-linked networks, the situation is somewhat more difficult but a biexponential behavior remains if the Gaussian decay due to the spins in the immediate vicinity of the cross-links is not considered. However, a sol fraction or residual contributions from the solvent itself give rise to a long, third component.

The transverse relaxation decay was observed with CPMG sequences of different pulse separation. A slow decay of about $2 \%$ in weight that was observed at all solvent concentrations but was absent from the dry rubber was attributed 
to the sol phase of uncross-linked compounds. The remaining two contributions to the signal decay are plotted in Fig. 6a for NR 5/5. While the long component increases steadily with increasing amount of solvent, the short component remains essentially unchanged. It must therefore be affected by the presence of the cross-links. In comparison, both components increase about proportionally for uncross-linked polyisoprene solutions of $\overline{M_{n}}=287000$, revealing the overall increase in mobility of the whole polymer chains (see Fig. 6b), i.e. the central and end parts of the chains are affected in the same way by the presence of the solvent. Transverse relaxation thus allows the identification of a component that is clearly determined by the restricting effect that cross-links must have on the ability of nearby chain segments to perform reorientations, while $T_{1}$ even at the lowest frequencies shows only a minor effect.

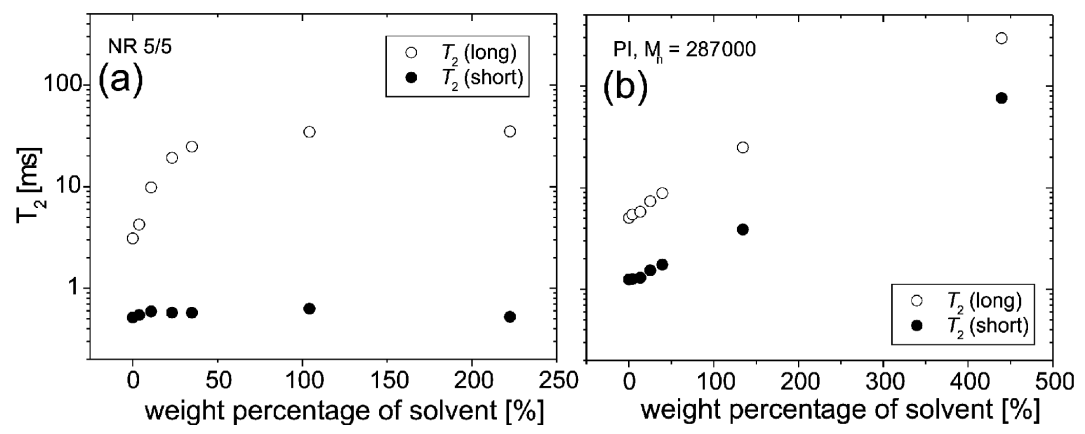

Fig. 6. (a) Short and long components of the ${ }^{1} H$ transverse relaxation time $T_{2}$ at a Larmor frequency of $200 \mathrm{MHz}$ for natural rubber NR $5 / 5$ at equilibrium swelling in ethanol/toluene mixtures. (b) Data obtained for solutions of polyisoprene of a number-averaged molecular weight of 287000 at the same relative amounts of solvent as in (a).

In much the same way, a distinction of the effect of cross-links on local order is revealed by measuring the dipolar coupling constant from the evolution of the double-quantum signal averaged over all proton pairs in the polyisoprene molecule. This quantity averages over motions acting on the time scale of the coupling constant itself. In Fig. 7a, a pronounced effect of the evolution (build-up and decay) of the double-quantum intensity is shown for polymer solutions, while the same measurements result in almost identical curves for the swollen networks. From the initial slope of the build-up curves, average coupling constants can be determined which are plotted in Fig. $7 \mathrm{~b}$ in comparison between solutions and swollen elastomers. Clearly, the order parameter tends toward zero if diluted solutions are regarded, while the presence of the cross-links maintains local order even if large amounts of solvent drastically increase the mobility of the chains themselves. Longitudinal relaxation, which senses this mobility but not the order itself, must therefore be regarded as an insufficient parameter for the characterization of molecular order. 


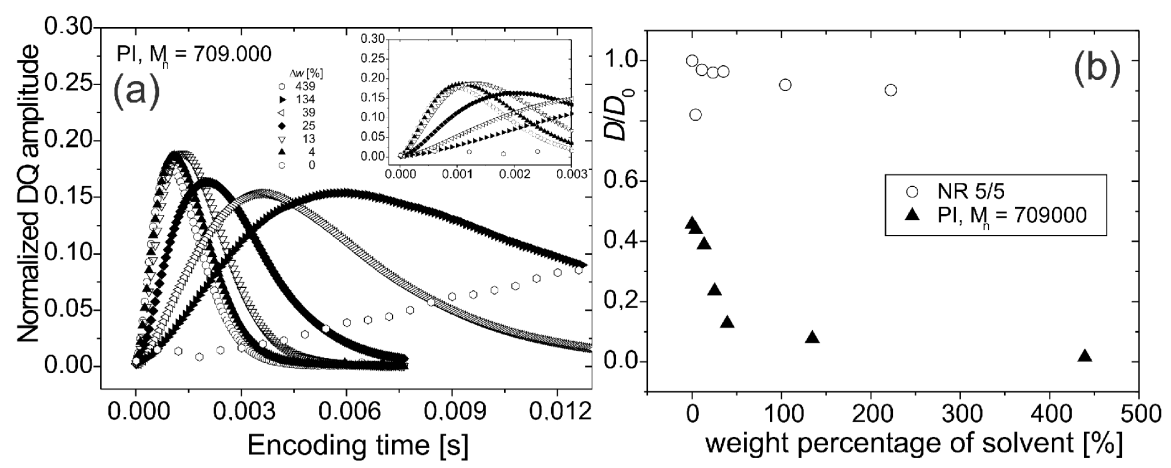

Fig. 7. (a) Double-quantum (DQ) built-up curves for the ${ }^{1} H$ signal in polyisoprene of a number-averaged molecular weight of 709000 dissolved in toluene. Numbers to the right of the plot indicate weight percentage of solvent relative to the dry polymer weight. The inset represent a magnification for short encoding times. (b) Relative dipolar coupling constants derived from the measurements in (a) compared to the results for natural rubber NR $5 / 5$ at equilibrium swelling as a function of weight percentage of solvent. Data are normalized to the coupling constant for NR $5 / 5$ in the absence of solvent, $D_{0}$.

\section{Conclusions}

Two possibilities for deforming elastomers were investigated, uniaxial stretching and swelling in a suitable solvent. Stretching was found to change the statistical distribution of segmental reorientations, leading to a steeper frequency dependence of the longitudinal relaxation time. In butadiene rubber where the relevant frequency range could be measured, an additional shift between different regions of dispersion was identified that was attributed to the more pronounced restrictions to motion in the local environment of the chain segments. Swelling, on the other hand, led to a dramatic increase in low-field relaxation times and an overall change of the dispersion towards Rouse behavior that is qualitatively very similar to solutions of uncross-linked chains. Transverse relaxation and homonuclear dipolar coupling strengths are much more sensitive to the presence of small concentrations of cross-links in the case of swelling. Partial deuteration of elastomers and the preparation of samples of much higher cross-link density can be considered as two major steps to reveal not only the statistical behavior but also the relative importance of individual interactions in the molecular dynamics of chain molecules under permanent chemical restriction.

\section{Acknowledgment}

The authors are grateful to Dr. Volker Herrmann from Degussa AG, Germany, to Dr. Klaus Unseld, Matthias Bode, and Thomas Böhner from Dunlop GmbH, Germany, for kindly providing the rubber samples, and to M. Adams, 
K. Kupferschläger, and G. Schroeder for technical support. Funding from Deutsche Forschungsgemeinschaft (Sta 511/3-1) is gratefully acknowledged.

\section{References}

[1] M. Doi, S.F. Edwards, The Theory of Polymer Dynamics, Clarendon, Oxford 1986.

[2] P.-G. de Gennes, J. Chem. Phys. 55, 572 (1971).

[3] K.S. Schweizer, J. Chem. Phys. 91, 5802 (1991).

[4] N. Fatkullin, R. Kimmich, H.W. Weber, Phys. Rev. E 47, 4600 (1993).

[5] N. Fatkullin, R. Kimmich, J. Chem. Phys. 101, 822 (1994).

[6] R. Kimmich, H.W. Weber, J. Chem. Phys. 98, 5847 (1993).

[7] H.W. Weber, R. Kimmich, Macromolecules 26, 2597 (1993).

[8] S. Kariyo, S. Stapf, Macromolecules 35, 9253 (2002).

[9] S. Kariyo, S. Stapf, Solid State Nucl. Magn. Reson. 25, 64 (2004).

[10] S. Kariyo, S. Stapf, B. Blümich, Macromol. Chem. Phys. 206, 1300 (2005).

[11] S. Kariyo, S. Stapf, Macromol. Chem. Phys. 206, 1291 (2005).

[12] R. Kimmich, K. Gille, N. Fatkullin, R. Seitter, S. Hafner, M. Müller, J. Chem. Phys. 107, 5973 (1997).

[13] H. Chaumette, D. Grandclaude, P. Tekely, D. Canet, C. Cardinet, E. Verschave, J. Phys. Chem. A 105, 8850 (2001).

[14] T.N. Khazanovich, Polymer Sci. USSR 4, 727 (1963).

[15] R. Kimmich, NMR: Tomography - Diffusometry - Relaxometry, Springer Verlag, Berlin 1997.

[16] E. Anoardo, G. Galli, G. Ferrante, Appl. Magn. Reson. 20, 365 (2001).

[17] R. Kimmich, E. Anoardo, Prog. NMR Spectr. 44, 257 (2004).

[18] R. Graf, D.E. Demco, S. Hafner, H.W. Spiess, Solid State Nucl. Magn. Reson. 12, 139 (1998).

[19] M. Schneider, L. Gasper, D.E. Demco, B. Blümich, J. Chem. Phys. 111, 402 (1999).

[20] R. Kimmich, N. Fatkullin, Adv. Polym. Sci. 170, 1 (2004).

[21] P.T. Callaghan, E.T. Samulski, Macromolecules 33, 3795 (2000).

[22] S. Murakami, K. Senoo, S. Toki, S. Kohjiya, Polymer 43, 2117 (2002).

[23] S. Toki, I. Sics, S. Ran, L. Liu, B.S. Hsiao, S. Murakami, K. Senoo, S. Kohjiya, Macromolecules 35, 6578 (2002).

[24] V.M. Litvinov, Macromolecules 34, 8468 (2001).

[25] A. Wiesmath, C. Filip, D.E. Demco, B. Blümich, J. Magn. Reson. 154, 60 (2002).

[26] R. Kimmich, N. Fatkullin, R.-O. Seitter, K. Gille, J. Chem. Phys. 108, 2173 (1998).

[27] R. Kimmich, M. Köpf, P.T. Callaghan, J. Polym. Sci. Polym. Phys. 29, 1025 (1991). 